\title{
EXTENSIONS OF COMMUTING ISOTONE FUNCTIONS ${ }^{1}$
}

\author{
BY FRANK W. OWENS
}

Communicated by D. Scott, June 6, 1966

The following problem was suggested as a research problem by Ralph De Marr in Bull. Amer. Math. Soc. 70 (1964), 501:

Let $A$ be a nonempty subset of the unit interval $I$. Let $f_{0}, g_{0}: A \rightarrow A$ be isotone functions (i.e., $f_{0}(x) \leqq f_{0}(y)$ if $\left.x \leqq y\right)$ such that $f_{0}\left(g_{0}(x)\right.$ ) $=g_{0}\left(f_{0}(x)\right)$ for all $x \in A$. Can $f_{0}$ and $g_{0}$ be extended to isotone functions $f, g: I \rightarrow I$ which still commute?

We shall show that the answer is yes under certain additional assumptions, and give a counterexample to the problem in the above form.

Definition. $A \subset I$ is called left (right)-closed if any decreasing (increasing) sequence in $A$ has a limit in $A$. We write $A^{L}\left(A^{R}\right)$ for the left (right)-closure of $A$.

Remark. $A$ is closed iff $A$ is left-closed and right-closed, i.e., $\bar{A}=A^{L} \cup A^{R}$.

Theorem 1. If $A \cup\{\inf A\}$ is left-closed or $A \cup\{\sup A\}$ is rightclosed, there exist commuting isotone extensions.

Proof. We give the proof for the case $A \cup\{\inf A\}$ is left-closed. The case $A \cup\{\sup A\}$ is right-closed is similar. Extend $f_{0}$ and $g_{0}$ to [0, inf $A] \cup A$ by defining them to be zero on [0, inf $A]$ if inf $A \notin A$, and to be their respective values at inf $A$ if $\inf A \in A$. Next extend $f_{0}$ and $g_{0}$ to $B=[0, \inf A] \cup A \cup[\sup A, 1]$ by defining them to be one on [sup $A, 1]$ if $\sup A \notin A$, and to be their respective values at $\sup A$ if $\sup A \in A$. Define $j: I \rightarrow B$ by $j(x)=\inf \{y \in B \mid x \leqq y\} . j$ is isotone on $I$, and $j \mid B=$ the identity function on $B$. The required extensions are $f=f_{0} j$ and $g=g_{0} j$. $f$ and $g$ are isotone since the composition of two isotone functions is isotone. $f_{0} j \mid A=f_{0}$ and $g_{0} j \mid A=g_{0} . f$ and $g$ commute on $I$ since $f_{0} j g_{0} j=f_{0} g_{0} j=g_{0} j_{0} j=g_{0} j f_{0} j$.

Note. The proof of the case $A \cup\{\sup A\}$ is right-closed is the same except that we define $j: I \rightarrow B$ by $j(x)=\sup \{y \in B \mid y \leqq x\}$.

Definition. Let $h: A \rightarrow A$ be isotone. Define $h^{L}: A^{L} \rightarrow A^{L}$ and $h^{R}: A^{R} \rightarrow A^{R}$ by

$$
\begin{aligned}
h^{L}(x) & =h(x), & & x \in A \\
& =\inf \{h(y) \mid x \leqq y \in A\}, & & x \in A^{L}-A
\end{aligned}
$$

\footnotetext{
${ }^{1}$ Partially supported by N. S. F. Grant GP-5855.
} 
and

$$
\begin{aligned}
h^{R}(x) & =h(x), & & x \in A \\
& =\sup \{h(y) \mid x \geqq y \in A\}, & & x \in A^{R}-A .
\end{aligned}
$$

REMARK. $h^{L}$ and $h^{R}$ are well defined. $h^{L}$ is right continuous on $A^{L}$ if $h$ is right continuous on $A$, and $h^{R}$ is left continuous on $A^{R}$ if $h$ is left continuous on $A$.

THEOREM 2. If $f_{0}$ and $g_{0}$ are both right or left continuous on $A$, there exist commuting isotone extensions.

OUtLINe of Proof. $f_{0}^{L}$ and $g_{0}^{L}\left(f_{0}^{R}\right.$ and $\left.g_{0}^{R}\right)$ commute on $A^{L}\left(A^{R}\right)$ if $f_{0}$ and $g_{0}$ are right (left) continuous on $A$. Apply Theorem 1 to get $f_{0}^{L} j$ and $g_{0}^{L} j$ ( $f_{0}^{R} j$ and $g_{0}^{R} j$ ) for the required extensions.

These and similar theorems can be generalized to complete lattices.

We shall now give a counterexample to the problem in its weak form. Let $A=[0,1 / 2) \cup(1 / 2,1]$. Define $f_{0}$ and $g_{0}$ by

$$
\begin{aligned}
& f_{0}(x)=3 / 4 \quad \text { for } \quad 0 \leqq x \leqq 3 / 8 \\
& =4 x / 3+1 / 4 \text { for } 3 / 8 \leqq x<1 / 2 \\
& =11 / 12 \text { for } 1 / 2<x \leqq 3 / 4 \\
& =1 \quad \text { for } 3 / 4<x \leqq 1 \text {, }
\end{aligned}
$$

and

$$
\begin{array}{rlrlrl}
g_{0}(x) & =3 / 4 & \text { for } & 0 \leqq x & <1 / 2 \\
& =x+1 / 4 & \text { for } & 1 / 2 & <x \leqq 2 / 3 \\
& =11 / 12 & \text { for } & 2 / 3 \leqq x & <11 / 12 \\
& =1 & & \text { for } & & 11 / 12 \leqq x \leqq 1 .
\end{array}
$$

$f_{0}, g_{0}: A \rightarrow[3 / 4,1] \subset A$ are isotone functions which commute on $A\left(f_{0}\left(g_{0}(x)\right)=g_{0}\left(f_{0}(x)\right)=11 / 12\right.$ for $0 \leqq x<1 / 2$, and $f_{0}\left(g_{0}(x)\right)=g_{0}\left(f_{0}(x)\right)$ $=1$ for $1 / 2<x \leqq 1)$. The unique isotone extensions $f$ and $g$ of $f_{0}$ and $g_{0}$ are defined at $x=1 / 2$ by $f(1 / 2)=11 / 12$ and $g(1 / 2)=3 / 4$. But $f(g(1 / 2))=f(3 / 4)=11 / 12$ and $g(f(1 / 2))=g(11 / 12)=1$.

STANFORD UNIVERSITY 